\title{
A Transgenic Mouse Model for Inducible and Reversible Dysmyelination
}

\author{
Carole Mathis, Colette Hindelang, Marianne LeMeur, and Emiliana Borrelli \\ Institut de Génétique et de Biologie Moléculaire et Cellulaire, Institut National de la Santé et de la Recherche \\ Médicale/Centre National de la Recherche Scientifique/Université Louis Pasteur, 67404 IIlkirch Cedex, \\ Communauté Urbaine de Strasbourg, France
}

\begin{abstract}
Oligodendrocytes are glial cells devoted to the production of myelin sheaths. Myelination of the CNS occurs essentially after birth. To delineate both the times of oligodendrocyte proliferation and myelination, as well as to study the consequence of dysmyelination in vivo, a model of inducible dysmyelination was developed. To achieve oligodendrocyte ablation, transgenic animals were generated that express the herpes virus 1 thymidine kinase (HSV1-TK) gene under the control of the myelin basic protein (MBP) gene promoter. The expression of the MBP-TK transgene in oligodendrocytes is not toxic on its own; however, toxicity can be selectively induced by the systemic injection of animals with nucleoside analogs, such as FIAU [1-(2-deoxy-2-fluoro- $\beta-\delta$ arabinofuranosyl)-5-iodouracil]. This system allows us to control the precise duration of the toxic insult and the degree of ablation of oligodendrocytes in vivo.
\end{abstract}

We show that chronic treatment of MBP-TK mice with FIAU during the first 3 postnatal weeks triggers almost a total depletion of oligodendrocytes in the CNS. These effects are accompanied by a behavioral phenotype characterized by tremors, seizures, retarded growth, and premature animal death. We identify the period of highest oligodendrocytes division in the first 9 postnatal days. Delaying the beginning of FIAU treatments results in different degrees of dysmyelination. Dysmyelination in MBP-TK mice is always accompanied by astrocytosis. Thus, this transgenic line provides a model to study the events occurring during dysmyelination of various intensities. It also represents an invaluable tool to investigate remyelination in vivo.

Key words: oligodendrocyte; inducible dysmyelination; transgenic; HSV1-TK; MBP promoter; CNS
The oligodendrocytes and the Schwann cells perform the formation of the myelin sheaths surrounding axons in the CNS and peripheral nervous system (PNS), respectively. Defaults of this process lead to multiple sclerosis (MS) in humans, characterized by foci of demyelination of the CNS and a dramatic effect on nervous activity (Steinman, 1996). Multiple hypotheses explain how MS may arise, but the cause of this disease is still unclear. Gene mutations in some constituents of myelin result in debilitating disorders in humans (Pham-Dinh et al., 1991; Snipes et al., 1993). Interestingly, some of these naturally occurring mutations have also been isolated in mice. Studies of these mutants, together with targeted mutation of some key myelin genes in mice, has helped the understanding of the role of each major component in myelination (Popko et al., 1987; Readhead and Hood, 1990; Konat and Wiggins, 1992; Kagawa et al., 1994; Nave, 1994; Readhead et al., 1994; Griffiths, 1996). Animal models of demyelination have also been generated by chemical insults or viral infections (Hall, 1972; Blakemore, 1973; Yajima and Suzuki, 1979; Rodriguez, 1992; Miller et al., 1995). However, the bias of these models is that other cells or systems are likely to be affected in addition to glial cells.

\footnotetext{
Received June 9, 2000; revised Aug. 2, 2000; accepted Aug. 4, 2000.

This work was supported by funds from Centre National de la Recherche Scientifique, Institut National de la Santé et de la Recherche Médicale, Hôpitaux Universitaires de Strasbourg, the Association pour la Recherche sur le Cancer, and Mission Interministérielle à la Lutte contre la Drogue et la Toxicomanie (to E.B.). C.M. was supported by fellowships from the French Ministère de l'Education Nationale, de la Recherche et de la Technologie, and the Association pour la Recherche sur le Cancer. We acknowledge Dr. A. Giangrande, Dr. P. Sassone-Corsi, Dr. S. Tan, L. F. Collin and members of the laboratory for helpful discussion. We thank Dr. M. Kimura for the MBP promoter, Dr. P. Collins and Glaxo Wellcome for the anti-HSV1-TK antibody, and Bristol-Myers Squibb for generously providing FIAU. We are grateful to V. Heidt and E. Erbs for technical assistance, S. Falcone for animal care, B. Boulay and J. M. Lafontaine for artwork, and J. L. Vonesch and N. Messaddeq for image analyses.

Correspondence should be addressed to Emiliana Borrelli, Institut de Génétique et de Biologie Moléculaire et Cellulaire, Institut National de la Santé et de la Recherche Médicale/Centre National de la Recherche Scientifique/Université Louis Pasteur, Boîte Postale 163, 67404 Illkirch Cedex, Communauté Urbaine de Strasbourg, France. E-mail: eb@igbmc.u-strasbg.fr.

Copyright (C) 2000 Society for Neuroscience $0270-6474 / 00 / 207698-08 \$ 15.00 / 0$
}

The role of oligodendrocytes in both the support and maintenance of other cell types, as well as in the function of neurons, has not been established. Here we report the generation of a transgenic mouse model to study myelinogenesis by selective and temporal ablation of oligodendrocytes in vivo. Ablation was achieved using the herpes simplex virus 1 thymidine kinase (HSV1-TK) gene as a toxigene under the control of the mouse myelin basic protein (MBP) promoter. This system is based on the higher affinity of HSV1-TK with respect to the endogenous kinase, for nucleoside analogs, such as FIAU [1-(2-deoxy-2-fluoro- $\beta$ - $\delta$-arabinof uranosyl)5 -iodouracil]. Once expressed in proliferating cells, HSV1-TK is able to convert such drugs into nucleotides that are cytotoxic to mitotic cells. This method was validated previously in vivo to study cell lineage and the function of different cell types (Borrelli et al., 1988, 1989; Heyman et al., 1989; Bush et al., 1998, 1999; Rindi et al., 1999).

MBP-TK mice have been used to evaluate whether the absence of oligodendrocytes affects normal developmental processes and to study the dynamic process of myelination in the CNS. Interestingly, chronic FIAU treatment of these mice starting from birth results in a massive disappearance of myelinated areas and of oligodendrocytes, as well. This phenotype is accompanied by retarded growth, seizures, tremors, and death of the mice during the third week after birth. Several degrees of dysmyelination can be achieved by delaying the beginning of the treatment within the first $10 \mathrm{~d}$ of postnatal life. We show that oligodendrocyte proliferation mainly occurs during the first $9 \mathrm{~d}$ after birth. The MBP-TK mice can therefore mimic different levels of dysmyelination and constitute a valuable tool to study the process of progressive remyelination of the CNS.

\section{MATERIALS AND METHODS}

MBP-TK DNA construct. The MBP-TK construct was obtained by the fusion of the $1.3 \mathrm{~kb}$ HindIII $(-1317) / B a m H I ~(+35)$ fragment (Okano et al., 1988; Tamura et al., 1988; Campagnoni et al., 1993) of the mouse MBP promoter with the $1.5 \mathrm{~kb} \mathrm{BamHI} / \mathrm{NcoI}$ fragment of the HSV1-TK coding sequence (Borrelli et al., 1988).

Transgenic mice. The $2.8 \mathrm{~kb}$ Hin $\mathrm{dIII} / \mathrm{NcoI}$ fragment was injected into fertilized mouse oocytes from C57BL6 $\times$ SJL, and four founders were 
obtained. Founder 1 was chosen for analyses, and a transgenic line was established. In this line, $\sim 30$ copies of the transgene were integrated in the genomic DNA. The MBP-TK line is maintained by breeding transgenic females with C57BL6 males. Transgenic mice were genotyped by Southern blot analysis of genomic DNA isolated from tail biopsies. Blots were hybridized with a randomly primed fragment corresponding to the total HSV1-TK segment present in the transgene.

Injection of FIAU. The nucleoside analog FIAU was generously provided by Bristol-Myers Squibb (Wallingford, CT). FIAU was administered by daily subcutaneous injection of wild-type (WT) and transgenic mice from the same litter. The dose that achieves complete ablation of the oligodendrocytes is $40 \mathrm{mg} / \mathrm{kg}$ body weight.

Electron microscopy. After deep anesthesia, mice received an intracardiac perfusion of a $3 \%$ glutaraldehyde in $0.1 \mathrm{M} \mathrm{NaHPO}_{4}$ buffer, pH 7.4. Optic nerves and sciatic nerves were removed and kept overnight at $4^{\circ} \mathrm{C}$ in the same fixative. They were then post-fixed in $1 \%$ osmium tetroxide at $4^{\circ} \mathrm{C}$

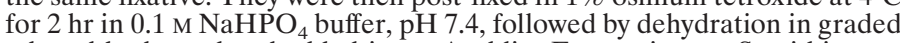
ethanol baths and embedded in an Araldite-Epon mixture. Semithin sections $(1 \mu \mathrm{m})$ were stained with toluidine blue. Ultrathin sections $(50 \mathrm{~nm})$ were contrasted with $5 \%$ uranyl acetate and lead citrate (Reynolds, 1963) and examined by electron microscopy (EM) (208 Philips EM).

In situ hybridization. Mouse brains were embedded in OCT (Tissue-Tek; Miles, Elkhart, IN) and frozen in dry ice. Sagittal and coronal sections (10 $\mu \mathrm{m})$ were cut on the cryostat, thaw-mounted onto gelatin-coated slides, and stored at $-80^{\circ} \mathrm{C}$ until hybridization. In situ hybridizations were performed as described previously (Saiardi et al., 1997). cDNA fragments for mouse MBP, myelin-associated glycoprotein (MAG), platelet-derived growth factor $\alpha$ receptor $(\mathrm{PDGF} \alpha \mathrm{R})$, glial fibrillary acidic proteins (GFAP), and HSV1-TK were subcloned into pBluescript SK ${ }^{-}$(Stratagene La Jolla, CA) to synthesize sense and antisense probes. ${ }^{35}$-labeled RNA probes were transcribed with either T7 or T3 RNA polymerase in the presence of cytidine $5^{\prime}-\alpha-\left[{ }^{35}\right.$ S $]$ thiotriphosphate $(10 \mathrm{mCi} / \mathrm{ml}$; Amersham Pharmacia Biotech, Little Chalfont, UK). After probe hybridization, slides were coated with Kodak NTB 2 emulsion (Eastman Kodak, Rochester, NY) and stored at $4^{\circ} \mathrm{C}$. The exposure time was $3 \mathrm{~d}$ for MBP, 1 month for GFAP, PDGF $\alpha$, and MAG riboprobes, and 2 months for HSV1-TK riboprobes. Emulsions were finally developed in Kodak 19 (Eastman Kodak), and tissues were counterstained with toluidine blue. Multiple labeling using digoxigenin-labeled riboprobes and antibodies were performed as described previously (Bernardoni et al., 1999). Histological analyses were performed on paraffin-embedded tissue after fixation in Bouin's fixative (Saiardi et al., 1997) using cresyl violet coloration.

Quantification of in situ hybridization. For each treatment, four animals from both genotypes were killed, and their brains were processed for in situ hybridization. Serial sagittal brain sections from transgenic and WT treated control mice were hybridized with a cRNA MBP probe and then exposed overnight directly in contact with an X-OMAT film (Eastman Kodak). Autoradiographs of all brain sections were finally scanned with an imaging densitometer apparatus (Bio-Rad, Hercules, CA) and quantified using the molecular Analyst software (Bio-Rad). The average level of MBP expression in WT animal brains was arbitrarily taken as $100 \%$ and was always compared with the corresponding values from the treated transgenic mice. Statistical analyses were performed using the Student's $t$ test

Cell counts. The proportion of cells in the CNS that are PDGF $\alpha$ Rpositive (PDGF $\alpha \mathrm{R}^{+}$) was estimated from bright-field micrographs of lightly toluidine blue-stained sections of thalamus. PDGF $\alpha \mathrm{R}^{+}$cells and the total number of cell nuclei were counted. At least five sections from each animal were analyzed. Approximately 500 total cells were counted in each section, and the proportion of $\mathrm{PDGF} \alpha \mathrm{R}^{+}$cells (mean $\pm \mathrm{SD}$ percentage) were tabulated (see Fig. 6).

Immunohistology. Transverse cryosections of brains from WT and MBPTK-treated mice were used for immunofluorescence study. The sections were post-fixed by immersion in Formalin (Sigma, Poole, UK) for $15 \mathrm{~min}$. The sections were preincubated for $1 \mathrm{hr}$ in $5 \%$ normal goat serum and $0.05 \%$ Tween 20 in PBS at room temperature, followed by incubation with primary antibodies (at the appropriate dilution) at $4^{\circ} \mathrm{C}$ overnight. Slides were then incubated $1 \mathrm{hr}$ with secondary antibodies. Controls for the specificity of each antibody were obtained by incubations of sections only with the secondary antibodies. Antibody dilutions were as follows: rabbit anti-GFAP (Sigma), 1:800; rabbit anti-HSV1-TK (a generous gift of Dr. P. Collins, Glaxo Wellcome, Beckenham, UK), 1:1000; mouse anti-O4 (Boehringer Mannheim, Mannheim, Germany), 1:5; proteolipid protein (PLP) (Chemicon, Temecula, CA), 1:800; galactocerebroside (GalC) (Boehringer Mannheim), 1:50; goat anti-rabbit conjugated with Cy3 (Jackson ImmunoResearch, West Grove, PA), 1:400; and goat anti-mouse conjugated with FITC (Jackson ImmunoResearch), 1:400. Phosphorylated histone 3 antibodies (H3P) (Upstate Biotechnology, Lake Placid, NY) and mouse anti-digoxigenin (Boehringer Mannheim) were used at 1:5000 and 1:1000, respectively. Immunolabeled sections were examined with a conventional microscope (Axiophot; Zeiss, Oberkochen, Germany) or with a confocal microscope (DMRE; Leica, Nussloch, Germany).

Northern blot analysis. Total RNA from cerebellum, pons, and cerebral hemispheres of WT and transgenic treated animals was prepared as described previously (Auffray et al., 1980). Five micrograms of total RNA was electrophoresed through $1 \%$ agarose $-4 \%$ formaldehyde gels in $10 \mathrm{~mm}$ sodium phosphate buffer and transferred to Hybond $\mathrm{N}+$ (Amersham
Pharmacia Biotech). Filters were hybridized with ${ }^{32} \mathrm{P}$-labeled DNA probes encoding mouse MBP, mouse PLP, rat cyclic 2,3-nucleotide phosphodiesterase (CNP), PDGF $\alpha$ R, and HSV1-TK. The human glucose 6-phosphate dehydrogenase (G6PD) was used as an internal loading control. Filters were washed and exposed to Kodak X-OMAT film (Eastman Kodak) for autoradiography. Quantification was performed using the Fuji (Tokyo, Japan) Bio-Imaging Analyzer BAS 2000.

Western blot analysis. Brains from 3-week-old WT and transgenic animals were rapidly dissected, frozen in liquid nitrogen, and homogenized in $1 \mathrm{ml}$ of lysis buffer (5 mM EDTA, $10 \mathrm{~mm}$ Tris, pH7.5, $5 \mu \mathrm{g} / \mathrm{ml}$ PMSF, $1 \mathrm{~mm}$ $\mathrm{NaF}, 1 \mathrm{~mm} \mathrm{Na} \mathrm{VO}_{4}, 1 \mu \mathrm{g} / \mathrm{ml}$ leupeptin, and $1 \mu \mathrm{g} / \mathrm{ml}$ aprotinin). Supernatants were separated by SDS-PAGE and transferred to nitrocellulose membrane. Membranes were blocked in $3 \%$ skim milk in $1 \times$ PBS and $0.02 \%$ Tween 20 and incubated with primary antibodies. Primary antibodies were used at the following dilutions: mouse anti-GFAP, 1:100 (ICN Biochemicals, Montréal, Québec, Canada); anti- $\beta$-tubulin, 1:1000 (Boehringer Mannheim); and anti-HSV1-TK, 1:250 (Dr. P. Collins). Blots were then incubated with either horseradish peroxidase (HRP)-conjugated goat anti-rabbit $\operatorname{IgG}(1: 10000)$ or HRP-conjugated horse anti-mouse $\operatorname{IgG}(1$ : 10000). Signals were developed in enhanced chemiluminescence Western blotting detection reagents (Amersham Pharmacia Biotech).

\section{RESULTS}

\section{The MBP-TK mouse model}

To study myelination in vivo, we generated transgenic animals expressing an inducible toxic gene in which oligodendrocytes could be selectively eliminated. The approach was designed to induce toxicity at different postnatal developmental stages. To do so, the HSV1-TK gene was expressed under the control of the $1.3 \mathrm{~kb}$ fragment of the mouse MBP promoter in transgenic mice. It has been shown that mitotic cells expressing HSV1-TK, both in vitro and in vivo, can be selectively killed upon daily administration of nucleoside analogs, such as FIAU (Borrelli et al., 1988, 1989; Heyman et al., 1989). Previous studies on the MBP promoter showed that the $1.3 \mathrm{~kb}$ fragment is sufficient to direct the specific expression of the transgene in oligodendrocytes (Turnley et al., 1991; Yoshioka et al., 1991). Furthermore, the presence of the MBP protein was observed in mitotic oligodendrocytes in vitro (Fressinaud et al., 1993).

The MBP-TK chimeric construct was injected into fertilized mouse eggs, and four founders were generated. The results presented relate to animals derived from the founder 1 , but equivalent phenotypes were obtained with transgenics that originated from the other lines. To verify the presence of the transgene, Southern blot analyses of mouse genomic DNA was performed using a probe corresponding to the HSV1-TK sequence (Fig. $1 A$ ). Northern blot analysis of RNAs from different tissues revealed the specific presence of the MBP-TK transcript only in the brain (Fig. 1B). To further characterize the pattern of expression of the transgene, in situ hybridization analyses were conducted on brain sections from untreated transgenic mice. Serial sections were hybridized in parallel with TK and MBP riboprobes (Fig. 2). Localization of the TK mRNA was examined and compared with the pattern of expression of MBP mRNA. The results of these experiments clearly show the specific expression of the transgene in areas enriched in oligodendrocytes, such as the white matter tract of the cerebellum (Fig. 2). The TK expression perfectly matched MBP mRNA expression (Fig. $2 A, B$ ). In addition, experiments performed during MBP-TK mice postnatal development showed that the temporal appearance of the MBP-TK transcript coincided with that of MBP (data not shown). These results underscore the high fidelity of the expression pattern obtained in our mouse model and further demonstrate that the MBP promoter used here contains all of the regulatory information required for specific expression. In addition, these results also show that TK expression is not toxic to oligodendrocytes in untreated MBP-TK mice.

The presence of the HSV1-TK protein in transgenic mice was tested by Western blot of whole-brain extracts and by immunohistochemistry. A HSV1-TK band of the expected size was only detectable in transgenic animals (Fig. 1C). Double-stainings of oligodendrocytes with anti-HSV1-TK and the oligodendrocytespecific PLP and GalC antibodies (Ranscht et al., 1982) were performed. Examination with confocal microscopy showed that the TK staining colocalizes with both of the oligodendrocyte markers 


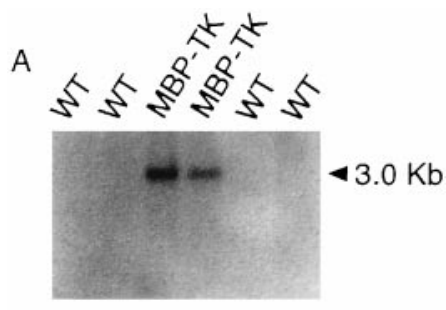

B

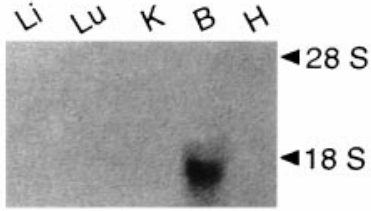

C

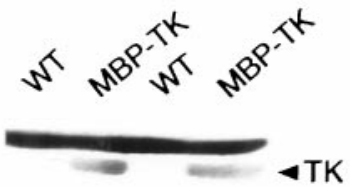

Figure 1. The MBP-TK transgene is specifically expressed in oligodendrocytes. $A$, Southern blot analysis of genomic DNA from tail biopsies. The HSV1-TK fragment was used as probe. $B$, Northern blot analysis of HSV1-TK expression in MBP-TK mice. Each lane contains $5 \mu \mathrm{g}$ of total RNA isolated from liver $(L i)$, lung $(L u)$, kidney $(K)$, brain $(B)$, and heart $(H)$. Same probe as $A$. $C$, Western blot analysis showing the presence of the HSV1-TK protein in the brain of adult transgenic mice. Protein extracts $(100 \mu \mathrm{g})$ from brains of adult WT and MBP-TK mice were used to detect the viral TK protein using a rabbit polyclonal antiserum raised against the HSV1-TK

but not with an astrocyte-specific protein, such as GFAP (Fig. 3A). Importantly, to allow toxicity using the TK system, cells must divide. Therefore, MBP mRNA should be present in oligodendrocytes that are still able to proliferate (Fig. $3 B$ ). To demonstrate this, we performed multiple staining of oligodendrocytes using a MBP digoxigenin-labeled riboprobe together with an antibody recognizing H3P. This experiment was made using sections of untreated MBP-TK 10-d-old transgenic mice. H3P has been shown to be a specific marker of dividing cells (Hendzel et al., 1997). Indeed, H3P-positive oligodendrocytes (Fig. 3B, H3P) expressing MBP mRNA (Fig. $3 B, M B P$ ) were found in the white matter tract. A nuclear localization of $\mathrm{H} 3 \mathrm{P}$ was confirmed by the concomitant 4',6'-diamidino-2-phenylindole (DAPI) staining (Fig. 3B,DAPI). $\mathrm{H} 3 \mathrm{P}, \mathrm{MBP}$, and DAPI staining colocalized in the same cells (Fig. $3 B, H 3 P / M B P / D A P I)$. As expected, in these same sections, we also observed MBP-positive H3P-negative oligodendrocytes.

\section{Dysmyelination in transgenic mice treated with FIAU}

It was reported that myelination occurs during the first 3 postnatal weeks (Carson et al., 1983; Foran and Peterson, 1992). Thus, to induce the ablation of oligodendrocytes in MBP-TK mice, litters generated by the mating of a transgenic female with a WT C57BL6 male were at first injected subcutaneously every day with $40 \mathrm{mg} / \mathrm{kg}$ body weight of FIAU starting at postnatal day 1 (P1) and continuing for $20 \mathrm{~d}$. This treatment resulted in retarded growth in all of the transgenic animals. These mice showed clear signs of dysmyelination starting at P10 characterized by tremors, ataxia, and seizures (Griffiths, 1996). The frequency and the degree of tonic seizures worsened with time and led to death at $\sim 3$ weeks after birth. Conversely, WT treated mice remained normal, demonstrating the specificity of action of FIAU in only the animals that carry the transgene. Similarly, untreated transgenics were completely normal.

Northern blot analysis was performed to quantify the reduction of oligodendrocytes by measuring the expression levels of RNAs that are specific markers of myelin (Campagnoni, 1988), such as $\mathrm{MBP}, \mathrm{CNP}$, and PLP in the cerebral hemispheres, the pons, and
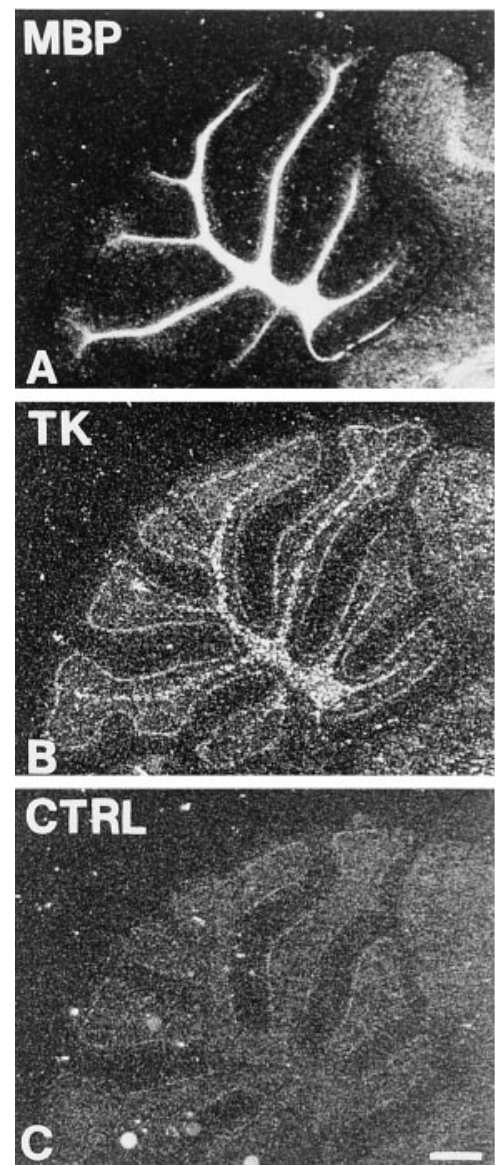

Figure 2. Overlapping expression patterns of the MBP-TK transgene and the endogenous MBP gene. Longitudinal adjacent cerebellar sections from an untreated 3-week-old MBP-TK mouse were hybridized with the MBP riboprobe $(A)$, the HSV1-TK riboprobe $(B)$, and the HSV1-TK sense riboprobe as control $(C)$. The labeling of Purkinje cells $(B, C)$ is nonspecific and results from the long exposure time for the TK antisense and sense probes ( 2 months). Scale bar, $400 \mu \mathrm{m}$.

the cerebellum (Fig. 4). In agreement with the described phenotype, a drastic $95 \%$ reduction of the MBP, PLP, and CNP mRNAs with respect to WT treated mice was observed. Thus, we have generated an inducible dysmyelination in the CNS of MBP-TKtreated mice.

To examine in greater detail the induced dysmyelination, in situ hybridization analysis was performed using the MBP probe and an additional marker of myelin, MAG. This analysis showed a homogeneous and drastic decrease of both mRNAs in the white matter of transgenic treated mice compared with WT (Fig. $5 A$ ). In agreement with the induced oligodendrocyte ablation (Fig. $5 B$ ), histological analysis on cresyl violet-stained sections showed an almost total absence of cell bodies in the corpus callosum of treated MBP-TK mice but not in WT treated littermates.

Importantly, Northern blot and in situ hybridization analyses using the PDGF $\alpha \mathrm{R}$ probe showed no difference in the expression of this marker between transgenic and WT treated mice. These results indicate that $\mathrm{PDGF} \alpha \mathrm{R}$-positive oligodendrocyte precursors are present in MBP-TK-treated mice and insensitive to FIAU treatment (Fig. 6). This is also in agreement with previously reported findings of absence of coincidental expression in oligodendrocytes of MBP and PDGF $\alpha$ R mRNAs (Butt et al., 1997).

In situ hybridization were also performed to assess the absence of nonspecific toxicity of FIAU treatments on different population of neurons. To do this, several markers were used (glutamic acid decarboxylase, substance P, enkephalin, p75, tyrosine hydroxylase, and zebrin) to compare their level of expression in WT and transgenic MBP-TK animals after treatment. No difference was found for all these markers between genotypes (data not shown). 

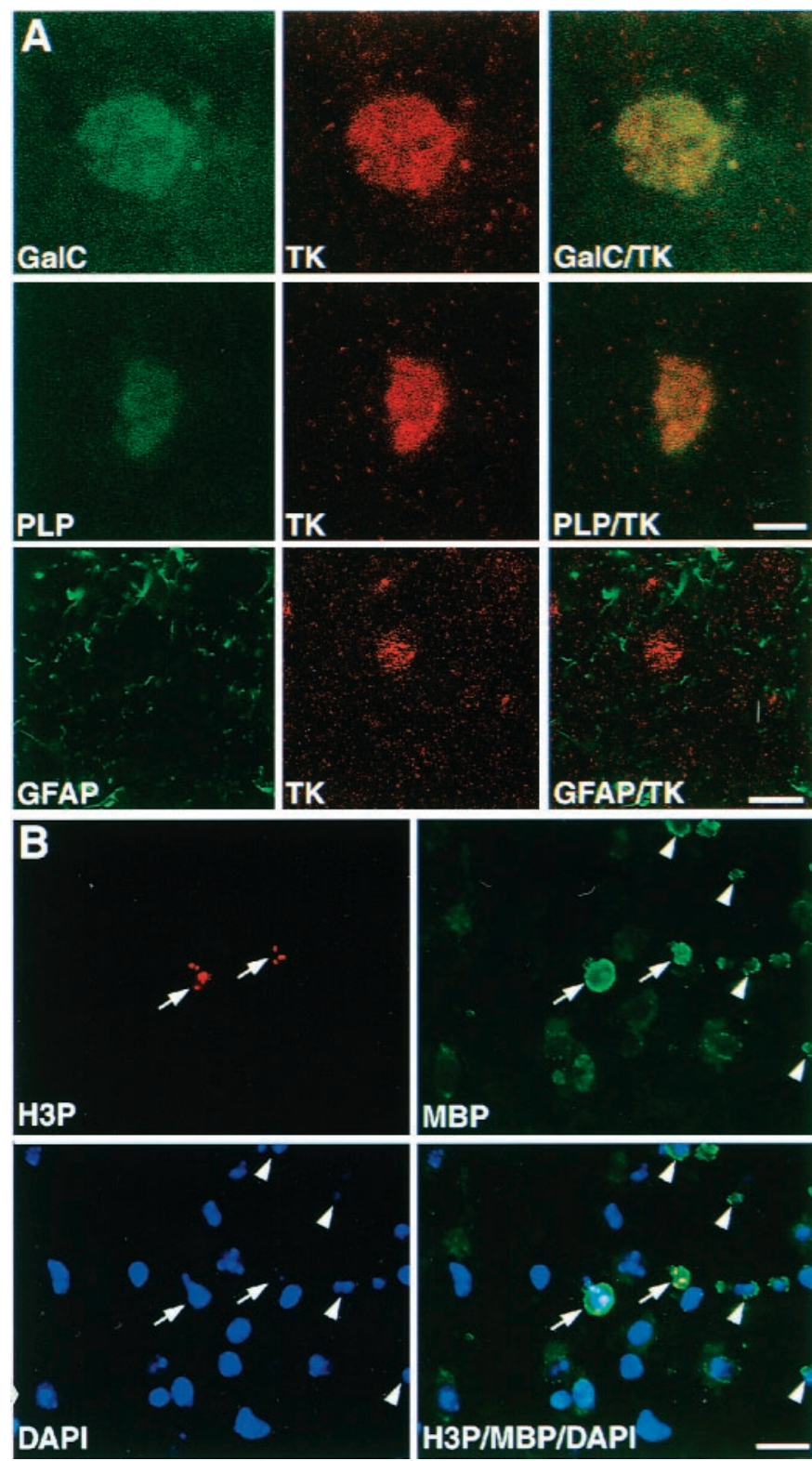

Figure 3. A, The HSV1-TK protein colocalizes with GalC and PLP but not with GFAP. Analyses were conducted by confocal microscopy on 3 -week-old brain sections from transgenic mice. Pictures represent single optical sections. TK immunostaining was revealed by $\mathrm{Cy} 3$-labeled secondary antibody and GalC and PLP with fluorescein. Small round individual cells immunoreactive for both HSV1-TK and GalC or PLP, portrayed in this figure, were taken in the pons at high magnification. No HSV1-TKpositive cells were stained by the mouse anti-GFAP. Scale bars: top two rows, $10 \mu \mathrm{m}$; bottom row, $40 \mu \mathrm{m}$. $B$, MBP mRNA is expressed by proliferating oligodendrocytes. Multiple-labeling of oligodendrocytes was performed on pons sections from a 10-d-old untreated transgenic mouse. MBP mRNA was visualized with a digoxigenin-labeled antisense riboprobe. Dividing cells were identified using a rabbit anti-H3P antibody. Nuclei were stained with DAPI. Arrows indicate dividing oligodendrocytes as shown by the colocalization of the MBP probe and the H3P staining. Arrowheads indicate, in the same field, postmitotic oligodendrocytes showing only MBP staining. Controls were performed using a MBP digoxigenin-labeled sense riboprobe, which did not stain cells in the pons. Scale bar, $10 \mu \mathrm{m}$.

\section{The MBP-TK transgene expression is restricted to the CNS}

Because MBP transcripts are present in Schwann cells (Gow et al., 1992), we also controlled whether myelination was affected within the PNS of transgenic mice. Transgenic and WT littermates, treated with FIAU as described, were killed, and the optic nerves and sciatic nerves (which belong, respectively, to the CNS and PNS) were dissected and processed for EM analyses. Importantly,
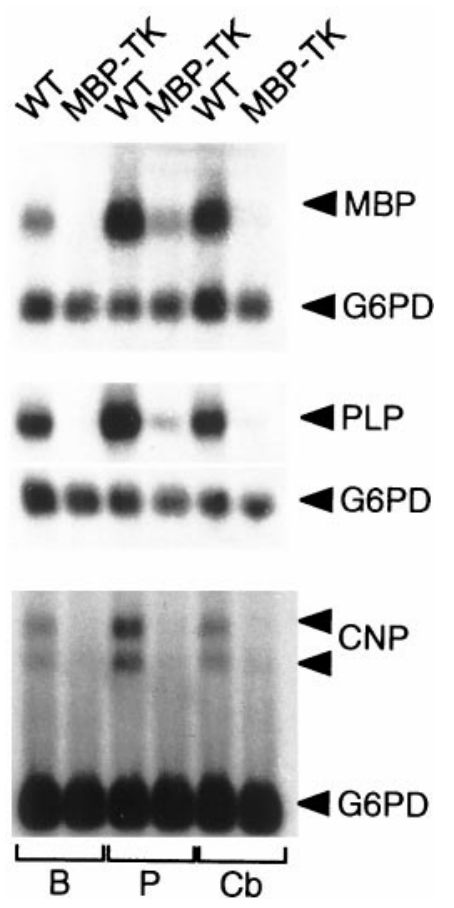

Figure 4. Northern blot analysis of mRNA expression of myelin markers in the brain of chronically treated $(1-20 \mathrm{~d}) \mathrm{WT}$ and transgenic siblings. Total mRNA $(5 \mu \mathrm{g})$ was used per lane. $B$, Brain; $P$, pons; $C b$, cerebellum. Blots were hybridized with MBP-, PLP-, and CNP-specific probes, as indicated. The G6PD probe was used as an internal standard of loaded quantities. The size of MBP, PLP, G6PD, and CNP bands is 2.3, 3.2, 1.2, and 2.4, $2.6 \mathrm{~kb}$, respectively.

no sign of dysmyelination was found in the sciatic nerves of either the WT or transgenic treated mice (Fig. $7 A, B$ ). These data confirmed our previous results on the selective function of HSV1-TK and again stress the highly cell-specific expression elicited by the proximal region of the MBP gene promoter that targets oligodendrocytes (Kimura et al., 1989; T urnley et al., 1991; Yoshioka et al., 1991; Foran and Peterson, 1992; Gow et al., 1992; Goujet-Zalc et al., 1993; Ikenaka and Kagawa, 1995).

Optic nerves from MBP-TK-treated mice were strongly dysmyelinated (Fig. 7C,D). Quantification of this data showed that $88 \%$ of the axons in the optic nerve of the transgenic treated mice were not myelinated. Nevertheless, some axons of the transgenic optic nerve were well myelinated with normal multilamellar, spirally wrapped sheaths of myelin around the nerve fibers. This indicates that some oligodendrocytes escaped the ablation, suggesting that they were postmitotic at the beginning of the treatment.

Interestingly, we noticed that the diameter of the nonmyelinated axons was smaller than that of the myelinated ones. This is in agreement with the notion that axon diameter growth is substantially reduced in the absence of oligodendrocytes (Colello et al., 1994; Sanchez et al., 1996) and suggests that the oligodendrocytes are able to regulate the diameter size of the optic axons.

Optic fiber numbers were determined throughout the length of WT and MBP-TK-treated nerves. After axon counts, we noticed that the total number of optic fibers in the transgenic treated mice was twofold higher than that in the WT myelinated optic nerves. These results support previous data (Colello and Schwab, 1994) and suggest that myelination has an inhibitory role on the sprouting of optic fibers.

\section{Identifying the key period for oligodendrocyte development}

Taking advantage of the TK ablation system, we decided to perform a number of different FIAU treatments (Fig. $8 B$ ) to delineate the phases of oligodendrocytes proliferation. Initially, four different treatments were conducted on MBP-TK mice and their WT sib- 


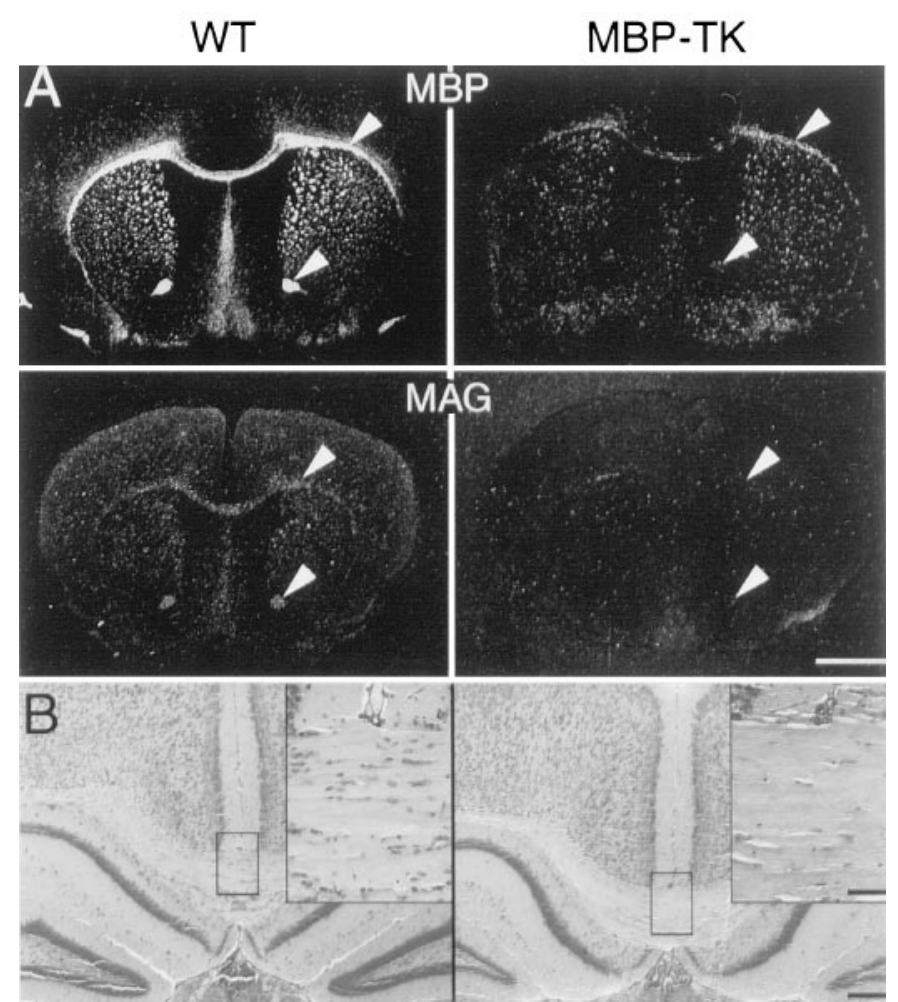

Figure 5. In situ hybridization analysis of MBP and MAG expression and histological examination of chronically treated (1-20 d) WT and MBP-TK mice. $A$, A drastic reduction of $\mathrm{MBP}$ and $\mathrm{MAG}$ expression is observed. Corpus callosum and anterior commissure are indicated by arrowheads. Scale bar, $1.45 \mathrm{~mm}$. $B$, Cresyl violet staining of paraffin-embedded brain sections from MBP-TK-treated and WT FIAU-treated mice showing a strong reduction in cell bodies specifically in the white matter tract of only MBP-TK-treated mice. Scale bar, $200 \mu \mathrm{m}$. The inset shows a higher magnification of the corpus callosum. Scale bar, $5 \mu \mathrm{m}$.

lings, which were daily injected subcutaneously from $24 \mathrm{hr}$ after birth and continued for $3(1-3 d), 6(1-6 d)$, or 9 d (1-9 d) with 40 $\mathrm{mg} / \mathrm{kg}$ body weight of FIAU. At P21, animals were killed, and brains were removed to be processed for histology, immunostaining, and gene expression analyses. The results of these studies clearly show a graded reduction of myelin in the CNS of treated transgenic animals as established by Luxol blue staining of brain sections from transgenic mice compared with WT animals (data not shown). To evaluate the extent of oligodendrocyte ablation, we performed in situ hybridization using the MBP probe. Densitometric scanning of autoradiograms revealed that treatment of MBP-TK mice during the first $3 \mathrm{~d}$ after birth did not result in a significant reduction of MBP expression (Fig. $8 C$ ). In contrast, a $50 \%$ reduction $(n=4)$ of MBP-specific signal was observed by extending the treatment to day 6 after birth. A drastic $95 \%$ reduction $(n=4)$ in MBP signal was seen when animals were treated for 9 consecutive days (Fig. 8). Importantly, the level of MBP expression in this last treatment did not differ significantly from that obtained in animals treated from day 1 to 20 (Fig. 4). Interestingly, both treatments (1-9 and 1-20 d) were characterized by the strongest behavioral phenotype, which included retarded growth, seizures, tremors, and death after the third week of age. These results strongly indicate that the bulk of oligodendrocyte proliferation takes place during the first 9 postnatal days. Similar results were also obtained by PLP immunostaining on brain sections (data not shown).

We then wondered whether the extent of oligodendrocyte ablation could be masked by proliferation of these cells after the arrest of the treatment. To solve this point, we conducted a parallel analysis with animals treated for 3, 6, 9, and $15 \mathrm{~d}$ and killed the day after the arrest. Quantification of MBP mRNA reduction in these mice $(10 \%$ at $3 \mathrm{~d}, 52 \%$ at $6 \mathrm{~d}, 90 \%$ at $10 \mathrm{~d}$, and $94 \%$ at $15 \mathrm{~d})$ gave
A
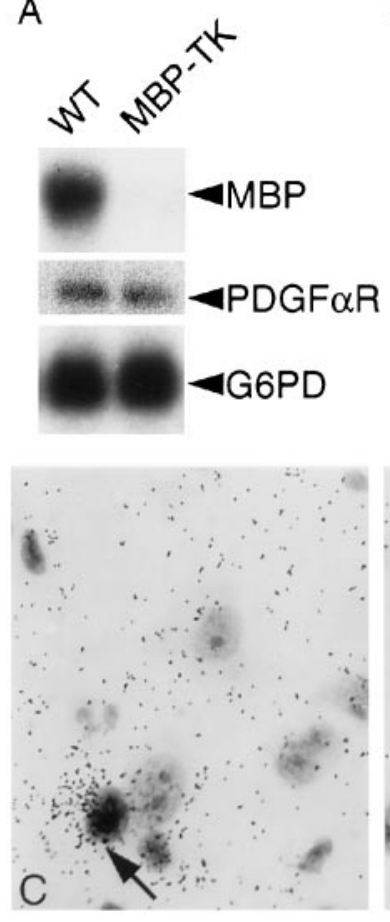

B
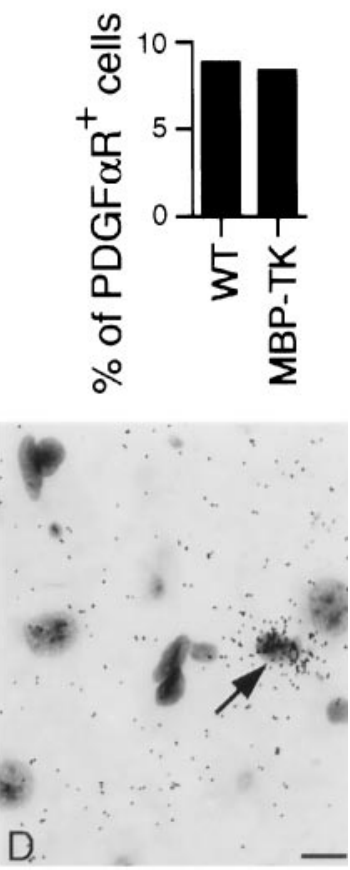

Figure 6. PDGF $\alpha$ R-positive oligodendrocytes are preserved in treated MBP-TK mice. $A$, Northern blot analysis of $5 \mu \mathrm{g}$ of total mRNA from brains of WT and transgenic 1- to 20-d-treated mice. Three different probes were used: MBP, PDGF $\alpha$ R, and G6PD. The size of MBP, PDGF $\alpha$ R, and G6PD bands is 2.3, 7, and $1.2 \mathrm{~kb}$, respectively. PDGF $\alpha$ R mRA expression level is not changed in the brain of transgenic compared with WT siblings, despite a strong reduction in MBP mRNAs. $B$, Total cell numbers were estimated, and PDGF $\alpha \mathrm{R}^{+}$cells were counted on the same sections in bright-field micrographs of in situ autoradiographs of the thalamus of WT and MBP-TK-treated (1-20 d) mice. Sections were stained with toluidine blue. Approximately 1500 total cells in at least five different sections were scored for each genotype, and the number of $\mathrm{PDGF} \alpha \mathrm{R}^{+}$cells are expressed as a percentage of the total (mean \pm SD percentage; WT, $8.96 \pm$ 0.8 ; MBP-TK, $8.8 \pm 1.2$ ). No significant difference (Student's $t$ test; $p>0.9$ ) was observed between genotypes. $C, D$, Higher magnification of bright-field micrographs of in situ autoradiographs with PDGF $\alpha \mathrm{R}$ antisense riboprobe of the thalamus region from WT $(C)$ and MBP-TK- $(D)$ treated $(1-20 \mathrm{~d})$ mice. Exposed silver grains are associated with cells with small, densely stained nuclei (arrows). Scale bar, $6.25 \mu \mathrm{m}$.

results analogous with those in which animals were killed at $21 \mathrm{~d}$. Interestingly, we noticed a $10 \%$ reduction in mice killed at $3 \mathrm{~d}$ that was not observed at $21 \mathrm{~d}$ in the 1-3 d treatment. This difference might be attributable to replacement of the ablated oligodendrocytes taking place after the injection period. With this exception, the result obtained with the other treatments suggest that a fixed number of oligodendrocytes is present in the postnatal brain and that, once ablated, the presence of these cells cannot be compensated.

Another important question is whether oligodendrocyte proliferation is a continuous process or if it proceeds in waves. To address this question, the beginning of FIAU treatments was delayed in time. Thus, animals were injected starting from day 3 (3-20 d), $6(6-20$ d), or $9(9-20$ d) after birth until day 20 and then killed on day 21 as in the other experiments. Analysis was performed at the histological and gene expression level. Quantification of in situ hybridization experiments showed the strongest reduction of MBP expression $(85 \%, n=4)$ in animals treated from day 3 to 20 (Fig. $8)$. Interestingly, animals that were treated from day 6 to 20 showed a 50.6\% reduction of MBP expression comparable with the reduction obtained in mice treated from day 1 to 6 (Fig. 8). Based on the notion that TK ablation occurs only in dividing cells, these results suggest that, $6 \mathrm{~d}$ after birth, one-half of the oligodendrocyte population is already postmitotic. In agreement, only an $18.8 \%(n=4)$ reduction of $\mathrm{MBP}$ expression was observed in mice treated from 


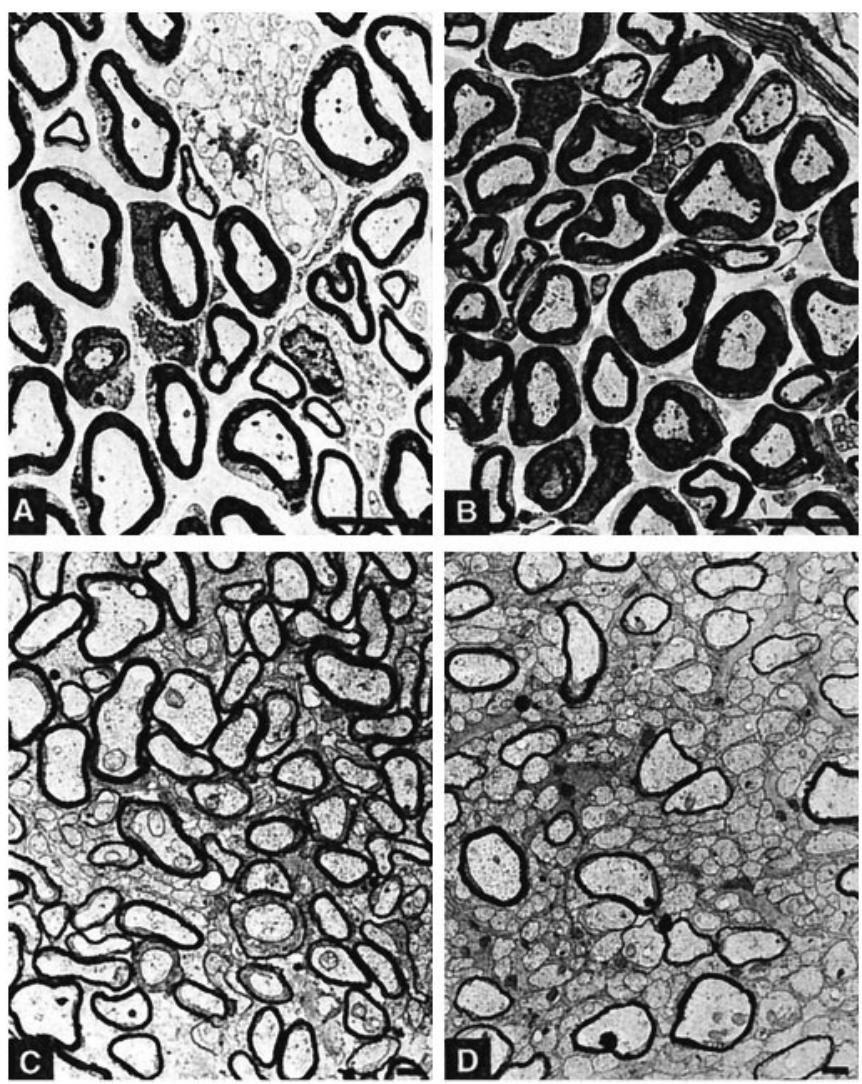

Figure 7. Electron microscopy analysis of myelination. The sciatic $(A, B)$ and optic $(C, D)$ nerves of WT $(A, C)$ and transgenic $(B, D)$ animals chronically treated with FIAU $(1-20 \mathrm{~d})$ were processed for EM analysis. Myelin loss is observed only in the optic nerve. Scale bars: $A, B, 1 \mu \mathrm{m} ; C$, $D, 0.1 \mu \mathrm{m}$.

day 9 to 20. These data indicate that the majority of proliferating oligodendrocytes are present during the first $10 \mathrm{~d}$ after birth. Histological analysis confirmed these results (data not shown).

\section{Expression of GFAP in the MBP-TK-treated mice}

Reactive astrocytosis is a characteristic phenomenon observed in response to various types of neurodegenerative disorders, such as MS, as well as in a wide range of other dysmyelinating diseases (Hatfield and Skoff, 1982; Li and Bartlett, 1991; Chen et al., 1993; Eddleston and Mucke, 1993). The hallmark of reactive gliosis is the increased expression of GFAP, the best characterized marker of astrocytes (Malhotra et al., 1990; Hoke and Silver, 1994; Ridet et al., 1997). In situ hybridization and immunohistochemical analyses of GFAP expression was performed in the CNS of MBP-TK and WT mice undergoing all the treatments aforementioned. In situ hybridization experiments revealed increased GFAP expression throughout the brain of FIAU-treated transgenic animals from day 1 to 20 and from day 1 to 9 , whereas no signs of astrocytosis were observed in WT treated siblings (data not shown). In these animals, quantification of both in situ hybridization and Northern blot analyses showed a similar increased level of GFAP mRNA (40$50 \%$ ) in all regions of the brain of dysmyelinated animals. Immunofluorescence studies supported these findings and revealed the presence of reactive astrocytes characterized by hypertrophic extensions strongly GFAP-positive in the transgenic treated mice (Fig. 9).

Clear signs of astrocytosis were already observed in the pons in 6-d-old mice chronically treated (data not shown). Thus, myelin loss induced in MBP-TK mice leads to reactive astrogliosis, as previously observed in other models of dysmyelinating diseases (Hatfield and Skoff, 1982; Li and Bartlett, 1991; Chen et al., 1993; Eddleston and Mucke, 1993).
A
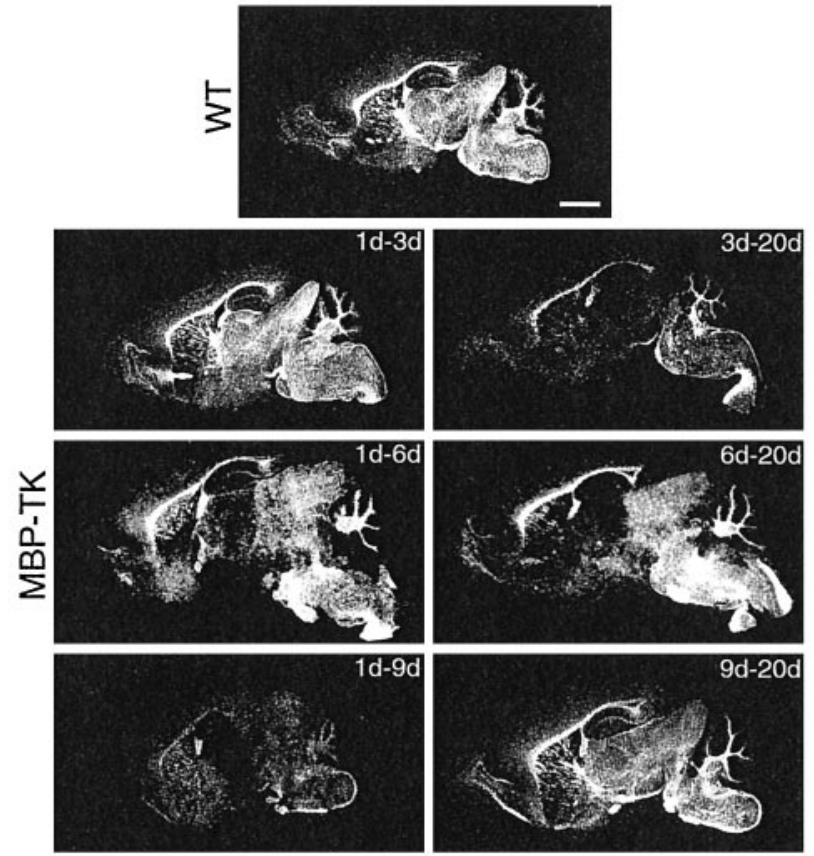

B

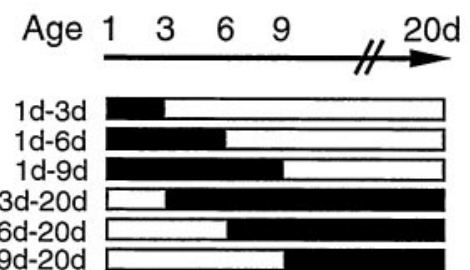

FIAU Treatment

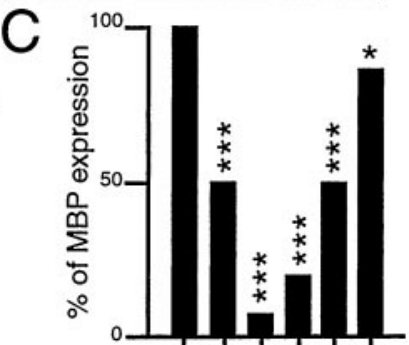

1
0
0
0
0
0
0
Figure 8. Inducible degrees of dysmyelination in MBP-TK mice. $A$, MBP in situ hybridization of sagittal brain sections from FIAU-treated 21-d-old WT and MBP-TK siblings. The length of the treatment is indicated. Scale bar, $2 \mathrm{~mm}$. $B$, Schedule of the FIAU protocols $(40 \mathrm{mg} / \mathrm{kg}$ body weight of FIAU) used to treat MBP-TK litters. $C$, Quantification of MBP in situ hybridizations. Densitometric analyses of whole-brain sections $(n=160$ sections from 4 different mice per genotype per treatment) for the different treatments are represented. The average level of MBP expression in age and treatment matched WT brain was arbitrarily taken as $100 \%$. Values represent mean \pm SEM percentage: $1-3 \mathrm{~d}, 100 \pm 0.7 ; 1-6 \mathrm{~d}, 52.3 \pm 0.23$; $1-9 \mathrm{~d}, 5 \pm 0.09 ; 3-20 \mathrm{~d}, 15.2 \pm 0.13 ; 6-20 \mathrm{~d}, 50.6 \pm 0.1$; and $9-20 \mathrm{~d}, 81.2 \pm$ 0.58 . Data were analyzed by Student's $t$ test: *p $<0.02 ; * * * p<0.0001$.

\section{DISCUSSION}

Oligodendrocytes are glial cells devoted to the synthesis and assembly of myelin sheaths around the axons of the CNS. Defaults of myelination cause serious impairments of nervous functions. Different models of dysmyelinating or demyelinating diseases are available in laboratory animals (Popko et al., 1987; Readhead and Hood, 1990; Konat and Wiggins, 1992; Kagawa et al., 1994; Nave, 1994; Readhead et al., 1994; Griffiths, 1996). The majority of these are mouse models represented by spontaneous mutations in the genes encoding for some myelin components. These animal models have illustrated the importance of myelin components in the process of myelination. However, these models have the bias to be irreversible because the default is present in the animal from the very beginning of life. Other models have been obtained by chemical treatments or viral infections of animals (Hall, 1972; Blakemore, 1973; Yajima and Suzuki, 1979; Rodriguez, 1992; Miller et al., 1995); in this case, it is very hard to control at the same time the specificity, timing, and extent of the insult.

The mouse model described here bypasses all of these shortcomings. Indeed, we have been able to induce dysmyelination at various 

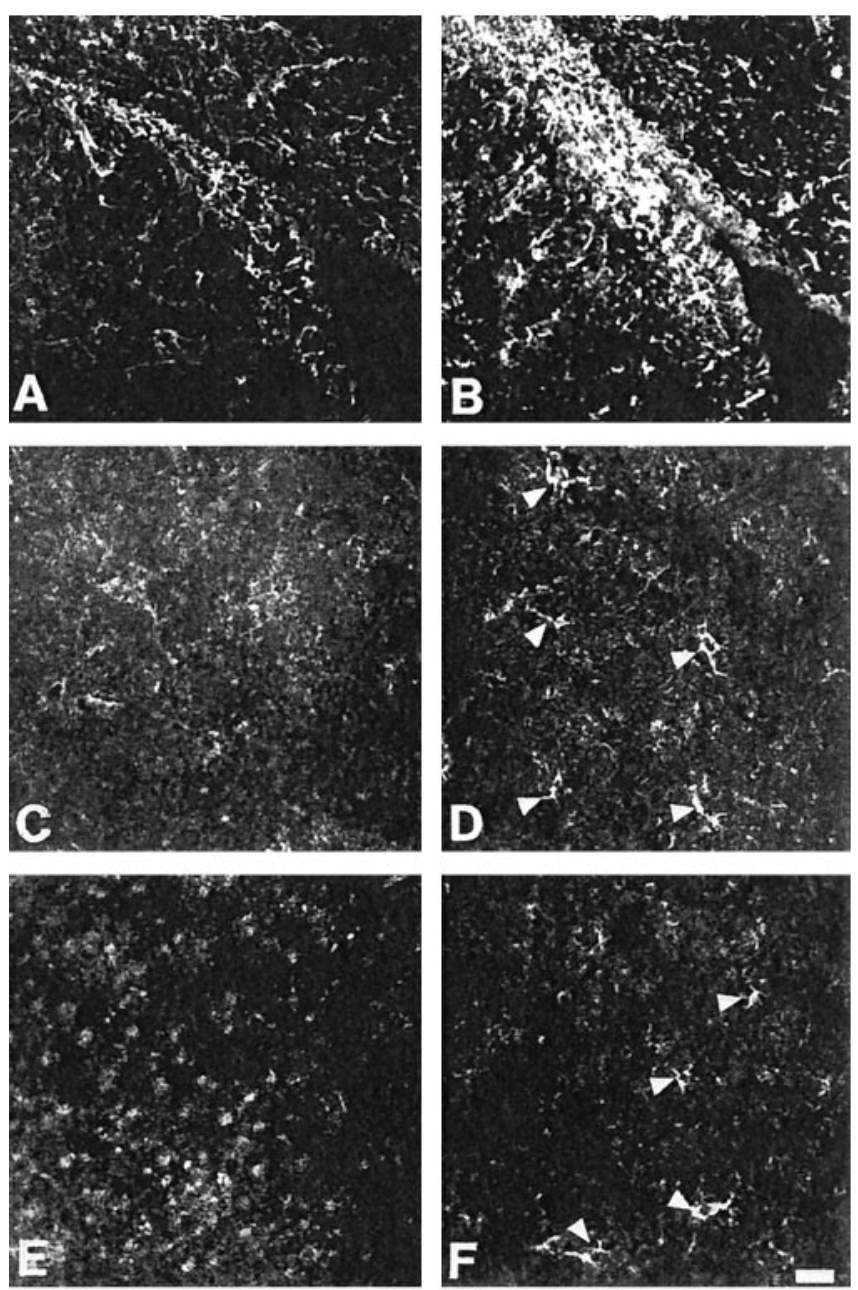

Figure 9. Astrocytosis in MBP-TK FIAU-treated mice. Immunohistochemical analyses were performed using a polyclonal anti-GFAP antibody. $A, C, E, \mathrm{WT} ; B, D, F$, MBP-TK. $A, B$, Subventricular zone; $C, D$, cortex; $E$, $F$, striatum. Scale bar, $100 \mu \mathrm{m}$.

intensities and for different lengths of time in a reproducible manner and in an established mouse line.

Importantly, we show that MBP expression is present in the first hours after birth and in oligodendrocytes that are still dividing. This finding seems to be in contrast with previous reports showing MBP expression mainly in postmitotic cells (Reynolds and Wilkin, 1991). However, here we present the evidence that MBP is expressed, at least at the mRNA level, when oligodendrocytes are still dividing (Fig. 3B). FIAU treatments selectively eliminate oligodendrocytes that are MBP-positive but not their precursors that are MBP-negative and PDGF $\alpha$ R-positive. No other cell types were directly affected by the FIAU treatment. The targeted function obtained with the MBP promoter shows that the expression of the transgene follows that of the endogenous MBP gene. More importantly, the fragment of promoter that we have used is not active in the PNS. This leads to the selective ablation of oligodendrocytes in the CNS.

Chronic daily treatment started $1 \mathrm{~d}$ after birth results in the most marked phenotype that is characterized by retarded growth, tremors, and seizures. A number of analyses of the CNS have shown nearly a total loss of myelin sheaths in the brain of treated transgenic animals, whereas the brains of treated WT littermates remain normal. These results show that it is possible to specifically kill oligodendrocytes only in the transgenic animals. Analyses of dysmyelination in the optic nerve of treated transgenics show that $85 \%$ of the axons are dysmyelinated. The size of these axons is reduced and their number is increased, well in agreement with previous data indicating that axon diameter is sensitive to myelin ensheathment (Colello et al., 1994; Sanchez et al., 1996). The increased number of optic fibers is indicative of sprouting, which has been described to take place in dysmyelinated rat optic nerves after x-ray exposure (Colello and Schwab, 1994).

Interestingly, the extent of dysmyelination is similar in the different areas of the brain, indicating that the bulk of myelinogenesis occurs during the first $9 \mathrm{~d}$ of postnatal development, following a caudorostral pattern. Indeed, treatments over the first $3 \mathrm{~d}$ of postnatal life did not result in a significant dysmyelination of the CNS. This indicates that the first days after birth do not correspond to a period of intense oligodendrocytes proliferation. Alternatively, it is plausible that this treatment did not result in a robust myelin depletion because the remyelination that takes place from day 3 to 21 could have masked the effect. In agreement with this, a reduction of MBP expression was observed in treated transgenic animals killed at $3 \mathrm{~d}$. However, when treatments were delayed to $6 \mathrm{~d}$ after birth, a stronger dysmyelination $(50.6 \%)$ was found in the cerebral hemispheres. Similarly, the treatment from day 1 to 9 resulted in a myelin reduction analogous to that achieved by treating the animals from day 1 to 20 . The importance of these results is twofold: (1) they indicate that the most intense period of oligodendrocyte proliferation occurs in the first $9 \mathrm{~d}$ after birth, and (2) that effective remyelination may occur only during these first $9 \mathrm{~d}$. After this period, the remyelinating processes must be much slower and are undetectable upon analysis of the mice at $21 \mathrm{~d}$ of age. This does not exclude the possibility that longer periods of time might be necessary to allow the rescue of the phenotype.

The MBP-TK mouse model of dysmyelination also presents a classical feature found in injuries of the CNS. Indeed, we have observed the presence of astrocytosis in all of the treatments that induce dysmyelination. This is characterized by increased GFAP staining and by the appearance of reactive astrocytes in brain regions that normally do not show any staining, such as the striatum and the cortex.

In conclusion, we believe that the MBP-TK mice represent an important addition to the already available models of diseases affecting myelinogenesis. This model of dysmyelination is very valuable because it is inducible and is not generated by a mutation in myelin-expressed genes. In addition, once the nucleoside treatment is arrested, it allows one to study the regenerative capacities of the system with normal cells. In particular, this is possible on animals treated with shorter protocols (i.e., 1-6 and 6-20 d) because they are viable after the treatment. Future studies will be aimed at analyzing the remyelination processes during longer periods of time and after different schedules. Similarly, the efficacy of compounds aimed at helping remyelination will also be tested.

\section{REFERENCES}

Auffray C, Rougeon F (1980) Purification of mouse immunoglobin heavy chain messenger RNAs total myeloma tumor RNA. Eur J Biochem 107:303-314.

Bernardoni R, Kammerer M, Vonesch JL, Giangrande A (1999) Gliogenesis depends on glide/gcm through asymmetric division of neuroglioblasts. Dev Biol 216:265-275.

Blakemore WF (1973) Demyelination of the superior cerebellar peduncle in the mouse induced by cuprizone. J Neurol Sci 20:63-72.

Borrelli E, Heyman R, Hsi M, Evans RM (1988) Targeting of an inducible toxic phenotype in animal cells. Proc Natl Acad Sci USA 85:7572-7576.

Borrelli E, Heyman RA, Arias C, Sawchenko PE, Evans RM (1989) Transgenic mice with inducible dwarfism. Nature 339:538-541.

Bush TG, Savidge TC, Freeman TC, Cox HJ, Campbell EA, Mucke L, Johnson MH, Sofroniew MV (1998) Fulminant jejuno-ileitis following ablation of enteric glia in adult transgenic mice. Cell 93:189-201.

Bush TG, Puvanachandra N, Horner CH, Polito A, Ostenfeld T, Svendsen CN, Mucke L, Johnson MH, Sofroniew MV (1999) Leukocyte infiltration, neuronal degeneration, and neurite outgrowth after ablation of scar-forming, reactive astrocytes in adult transgenic mice. Neuron 23:297-308.

Butt AM, Hornby MF, Kirvell S, Berry M (1997) Platelet-derived growth factor delays oligodendrocyte differentiation and axonal myelination in vivo in the anterior medullary velum of the developing rat. J Neurosci Res 48:588-596.

Campagnoni AT (1988) Molecular biology of myelin proteins from the central nervous system. J Neurochem 51:1-14. 
Campagnoni AT, Pribyl TM, Campagnoni CW, Kampf K, Amur-Umarjee S, Landry CF, Handley VW, Newman SL, Garbay B, Kitamura K (1993) Structure and developmental regulation of Golli-mbp, a 105-kilobase gene that encompasses the myelin basic protein gene and is expressed in cells in the oligodendrocyte lineage in the brain. J Biol Chem 268:4930-4938.

Carson JH, Nielson ML, Barbarese E (1983) Developmental regulation of myelin basic protein expression in mouse brain. Dev Biol 96:485-492.

Chen H, Cabon F, Sun P, Parmantier E, Dupouey P, Jacque C, Zalc B (1993) Regional and developmental variations of GFAP and actin mRNA levels in the CNS of jimpy and shiverer mutant mice. J Mol Neurosci 4:89-96.

Colello RJ, Schwab ME (1994) A role for oligodendrocytes in the stabilization of optic axon numbers. J Neurosci 14:6446-6452.

Colello RJ, Pott U, Schwab ME (1994) The role of oligodendrocytes and myelin on axon maturation in the developing rat retinofugal pathway. J Neurosci 14:2594-2605.

Eddleston M, Mucke L (1993) Molecular profile of reactive astrocytesimplications for their role in neurologic disease. Neuroscience 54:15-36.

Foran DR, Peterson AC (1992) Myelin acquisition in the central nervous system of the mouse revealed by an MBP-Lac Z transgene. J Neurosci 12:4890-4897.

Fressinaud C, Laeng P, Labourdette G, Durand J, Vallat JM (1993) The proliferation of mature oligodendrocytes in vitro is stimulated by basic fibroblast growth factor and inhibited by oligodendrocyte-type 2 astrocyte precursors. Dev Biol 158:317-329.

Goujet-Zalc C, Babinet C, Monge M, Timsit S, Cabon F, Gansmuller A, Miura M, Sanchez M, Pournin S, Mikoshiba K, Zalc B (1993) The proximal region of the MBP gene promoter is sufficient to induce oligodendroglial-specific expression in transgenic mice. Eur J Neurosci 5:624-632.

Gow A, Friedrich Jr VL, Lazzarini RA (1992) Myelin basic protein gene contains separate enhancers for oligodendrocyte and Schwann cell expression. J Cell Biol 119:605-616.

Griffiths IR (1996) Myelin mutants: model systems for the study of normal and abnormal myelination. BioEssays 18:789-797.

Hall SM (1972) The effect of injections of lysophosphatidyl choline into white matter of the adult mouse spinal cord. J Cell Sci 13:257-277.

Hatfield JS, Skoff RP (1982) GFAP immunoreactivity reveals astrogliosis in females heterozygous for jimpy. Brain Res 250:123-131.

Hendzel MJ, Wei Y, Mancini MA, Van Hooser A, Ranalli T, Brinkley BR, Bazett-Jones DP, Allis CD (1997) Mitosis-specific phosphorylation of histone $\mathrm{H} 3$ initiates primarily within pericentromeric heterochromatin during G2 and spreads in an ordered fashion coincident with mitotic chromosome condensation. Chromosoma 106:348-360.

Heyman RA, Borrelli E, Lesley J, Anderson D, Richman DD, Baird SM, Hyman R, Evans RM (1989) Thymidine kinase obliteration: creation of transgenic mice with controlled immune deficiency. Proc Natl Acad Sci USA 86:2698-2702.

Hoke A, Silver J (1994) Heterogeneity among astrocytes in reactive gliosis. Perspect Dev Neurobiol 2:269-274.

Ikenaka K, Kagawa T (1995) Transgenic systems in studying myelin gene expression. Dev Neurosci 17:127-136.

Kagawa T, Ikenaka K, Inoue Y, Kuriyama S, Tsujii T, Nakao J, Nakajima K, Aruga J, Okano H, Mikoshiba K (1994) Glial cell degeneration and hypomyelination caused by overexpression of myelin proteolipid protein gene. Neuron 13:427-442.

Kimura M, Sato M, Akatsuka A, Nozawa-Kimura S, Takahashi R, Yokoyama M, Nomura T, Katsuki M (1989) Restoration of myelin formation by a single type of myelin basic protein in transgenic shiverer mice. Proc Natl Acad Sci USA 86:5661-5665.

Konat G, Wiggins RC (1992) Genetic dysmyelination models. In: Neuromethods, Vol 21, Animal models of neurological disease I (Boulton A, Baker G, Butterworth R, eds), pp 175-195. Totowa, NJ: Humana.

Li XS, Bartlett WP (1991) Developmental expression of glial fibrillary acidic protein and glutamine synthetase mRNAs in normal and jimpy mice. Brain Res Mol Brain Res 9:313-317.

Malhotra SK, Shnitka TK, Elbrink J (1990) Reactive astrocytes: a review. Cytobios 61:133-160.
Miller DJ, Asakura K, Rodriguez M (1995) Experimental strategies to promote central nervous system remyelination in multiple sclerosis: insights gained from the Theiler's virus model system. J Neurosci Res 41:291-296.

Nave KA (1994) Neurological mouse mutants and the genes of myelin. J Neurosci Res 38:607-612.

Okano H, Tamura T, Miura M, Aoyama A, Ikenaka K, Oshimura M, Mikoshiba K (1988) Gene organization and transcription of duplicated MBP genes of myelin deficient (shi mld) mutant mouse. EMBO J 7:77-83.

Pham-Dinh D, Popot JL, Boespflug-Tanguy O, Landrieu P, Deleuze JF, Boue J, Jolles P, Dautigny A (1991) Pelizaeus-Merzbacher disease: a valine to phenylalanine point mutation in a putative extracellular loop of myelin proteolipid. Proc Natl Acad Sci USA 88:7562-7566.

Popko B, Puckett C, Lai E, Shine HD, Readhead C, Takahashi N, Hunt SW, Sidman RL, Hood L (1987) Myelin deficient mice: expression of myelin basic protein and generation of mice with varying levels of myelin. Cell 48:713-721.

Ranscht B, Clapshaw PA, Price J, Noble M, Seifert W (1982) Development of oligodendrocytes and Schwann cells studied with a monoclonal antibody against galactocerebroside. Proc Natl Acad Sci USA 79:2709-2713.

Readhead C, Hood L (1990) The dysmyelinating mouse mutations shiverer (shi) and myelin deficient (shimld). Behav Genet 20:213-234.

Readhead C, Schneider A, Griffiths I, Nave KA (1994) Premature arrest of myelin formation in transgenic mice with increased proteolipid protein gene dosage. Neuron 12:583-595.

Reynolds ES (1963) The use of lead citrate at high $\mathrm{pH}$ as an electron opaque stain in electron microscopy. J Cell Biol 17:208-211.

Reynolds R, Wilkin GP (1991) Oligodendroglial progenitor cells but not oligodendroglia divide during normal development of the rat cerebellum. J Neurocytol 20:216-224.

Ridet JL, Malhotra SK, Privat A, Gage FH (1997) Reactive astrocytes: cellular and molecular cues to biological function. Trends Neurosci [Erratum (1998) 21:80] 20:570-577.

Rindi G, Ratineau C, Ronco A, Candusso ME, Tsai M, Leiter AB (1999) Targeted ablation of secretin-producing cells in transgenic mice reveals a common differentiation pathway with multiple enteroendocrine cell lineages in the small intestine. Development 126:4149-4156.

Rodriguez M (1992) Central nervous system demyelination and remyelination in multiple sclerosis and viral models of disease. J Neuroimmunol 40:255-263.

Saiardi A, Bozzi Y, Baik JH, Borrelli E (1997) Antiproliferative role of dopamine: loss of D2 receptors causes hormonal dysfunction and pituitary hyperplasia. Neuron 19:115-126.

Sanchez I, Hassinger L, Paskevich PA, Shine HD, Nixon RA (1996) Oligodendroglia regulate the regional expansion of axon caliber and local accumulation of neurofilaments during development independently of myelin formation. J Neurosci 16:5095-5105.

Snipes GJ, Suter U, Shooter EM (1993) The genetics of myelin. Curr Opin Neurobiol 3:694-702.

Steinman L (1996) Multiple sclerosis: a coordinated immunological attack against myelin in the central nervous system. Cell 85:299-302.

Tamura T, Miura M, Ikenaka K, Mikoshiba K (1988) Analysis of transcription control elements of the mouse myelin basic protein gene in HeLa cell extracts: demonstration of a strong NFI- binding motif in the upstream region. Nucleic Acids Res 16:11441-11459.

Turnley AM, Morahan G, Okano H, Bernard O, Mikoshiba K, Allison J, Bartlett PF, Miller JF (1991) Dysmyelination in transgenic mice resulting from expression of class I histocompatibility molecules in oligodendrocytes. Nature 353:566-569.

Yajima K, Suzuki K (1979) Demyelination and remyelination in the rat central nervous system following ethidium bromide injection. Lab Invest 41:385-392.

Yoshioka T, Feigenbaum L, Jay G (1991) Transgenic mouse model for central nervous system demyelination. Mol Cell Biol 11:5479-5486. 